\title{
Comparison of the Degree of Shrinkage Under Air and Nitrogen Atmospheres by Laser Displacement Sensor
}

\author{
Kentaro Taki *and Ryui Yamada
}

\author{
School of Natural System, College of Science and Technology, University of \\ Kanazawa, Kakumamachi, Kanazawa, Ishikawa 920-1192, Japan \\ *taki@se.kanazawa-u.ac.jp
}

\begin{abstract}
Radical UV curable resin commonly shrinks during photopolymerization, which is difficult to avoid. This study developed an apparatus to measure the degree of shrinkage under nitrogen and air atmospheres. The apparatus consisted of a laser displacement sensor to detect the height of the spun-cast resin on a sapphire plate, two fused silica windows, a nitrogen purging line, and a UV light source. The initial and final thickness of a mixture of diurethane dimethacrylate/1-hydroxycyclohexyl phenyl ketone showed a linear relationship between 50 and $140 \mu \mathrm{m}$. The linear relationship was used to calculate the degree of shrinkage assuming an initial film thickness of $80 \mu \mathrm{m}$. The degree of shrinkage in nitrogen atmosphere was $1.3 \%$ greater than that in air. The relationship between the degree of shrinkage and UV intensity in the nitrogen atmosphere exhibited a single linear relation, while that in air showed two lines. UV intensities lower than $15 \mathrm{~mW} / \mathrm{cm}^{2}$ exerted a more significant influence on the degree of shrinkage.
\end{abstract}

Keywords: Shrinkage, Oxygen inhibition, Laser

\section{Introduction}

Radical UV curable resins typically shrink during photopolymerization. The degree of shrinkage ranges between 1 and $10 \%$, and depends on the number of moieties and curing conditions.

The polymerization shrinkage and related residual stress has been studied in the field of dental materials. The photopolymerization has been attracted since the bis-GMA-based composites was developed for the direct and indirect dental restoratives $[1,2]$. The volume shrinkage is also important issue of nanoimprint lithography for improving the demolding and final shape of nano/micro structures [3-8]. The stereolithography and other type of additive manufacturing using photopolymers is the layer-by-layer building process where the volume shrinkage should be controlled [9-12]. The primary study about the volume shrinkage based on the free volume theory was directed by the group of Bowman [13-15].

The measurement of shrinkage, residual stress, and conversion has been studied extensively over the past 20 years with the development of new instruments. Watts et al. developed the Bioman shrinkage stress device which enabled the measurement of the shrinkage displacement [16]. Lu et al. developed an apparatus which consisted of a cantilever and near infrared spectrometer to measure the degree of shrinkage, residual stress, and conversion simultaneously [17]. Neo and Park used a laser displacement sensor and ATR FT-IR (Attenuated Total Reflection Fourier Transform Infrared spectroscopy) technique to monitor the degree of shrinkage and conversion, simultaneously [18]. Jian introduced a simple laser displacement sensor to detect changes in film thickness to calculate the degree of shrinkage. This device is quite simple, although measurements of conversion and residual stress cannot be performed with this device [19]. Schmidt and Scherzer evaluated shrinkage using a hyphenated photorheometer and near-infrared spectroscopy [20]. Arenas et al. developed the interferometric technique to measure both local and global shrinkage phenomena [21].

Although the development of new devices has been adequate, the effect of atmosphere on the 
degree of shrinkage remains unclear. The degree of shrinkage in air is expected to be lower than under a nitrogen atmosphere [22]. The shrinking phenomena is related to the sequential phenomena of polymerization, cross-linking, and network formation.

In this study, an apparatus for measuring the degree of shrinkage under nitrogen and air atmospheres was developed to understand the effect of oxygen on the degree of shrinkage.

\section{Experimental}

\subsection{Materials}

A mixture of diurethane dimethacrylate (DUDM, Sigma-Aldrich) and 1-hydroxycyclohexyl phenyl ketone (Irgacure 184, BASF) with a 99:1 weight ratio was used as a UV-curable resin. The mixture was stirred at room temperature and stored for at least one night to ensure better homogeneity.

\subsection{Hybrid UV-LED}

The laser displacement sensor is simple and versatile for measuring the height of materials. To compare the degree of shrinkage under air and nitrogen-purged atmospheres, the laser displacement sensor was attached to an originallydeveloped optical apparatus, as shown in Fig. 1. The laser displacement sensor (LK-H008, laser wavelength of $655 \mathrm{~nm}$ ) was obtained from Keyence (Japan). The working distance was $8 \mathrm{~mm}$, applicable thickness was $\pm 0.5 \mathrm{~mm}$, and the minimum and maximum thickness that could be measured were 20 and $50 \mu \mathrm{m}$, respectively.

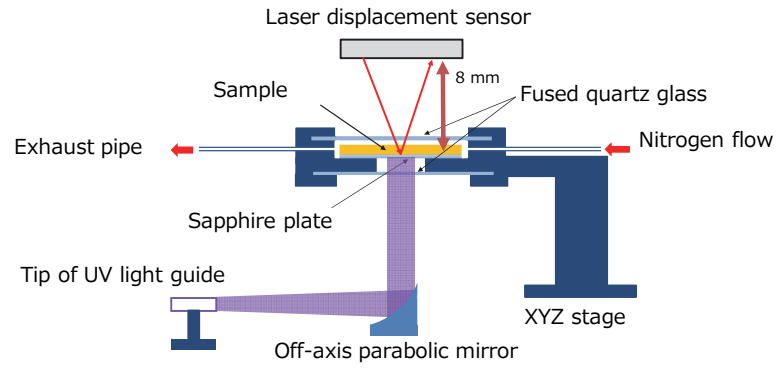

Fig. 1. Measurement apparatus for the degree of shrinkage.

The UV light source was a high-pressure mercury lamp (Omnicure S2000, ExFo). The UV light was passed through a liquid light guide $(\phi 5 \mathrm{~mm} \times 1500$ $\mathrm{mm}, \mathrm{P} / \mathrm{N}$ 805-00028, ExFo). The UV intensity was monitored with a UV meter which was sensitive to the $365 \mathrm{~nm}$ wavelength (UIT-150, USHIO, Japan). The UV light was collimated by an off-axis parabolic mirror (MPD129-F01, Thorlabs) with a reflected focal length of 2 inches. In the previous study [19], UV light was irradiated from $45^{\circ}$ above the sample. The UV light distribution was inhomogeneous, as shown in Fig. 2(a). However, even though a simple optical system, the off-axis parabolic mirror formed a uniform UV light distribution at the sample stage, as shown in Fig. 2(b).

The UV light distribution was captured using a band pass filter (360 nm, FB360-10, Thorlabs), fluorescence glass (excitation wavelength 200-420 $\mathrm{nm}$, fluorescence wavelength $610 \mathrm{~nm}$, Lumirace R7, Sumita optical glass, Japan), long pass filter (cut off wavelength $590 \mathrm{~nm}$, FGL590, Thorlabs), and a fixed-focal length lens (focal length $4.5 \mathrm{~mm}$, \#86900, Edmund) by the CMOS camera (DCC1545M, Thorlabs). The UV light exited the fluorescence glass and $610 \mathrm{~nm}$ fluorescence light was emitted and subsequently captured by the CMOS camera. The UV intensity distribution was determined from the intensity of the fluorescence light.

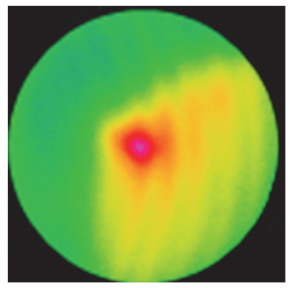

(a)

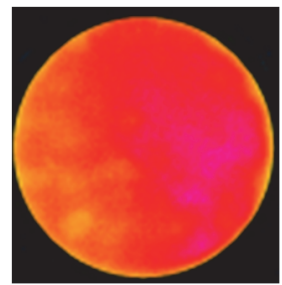

(b)
Fig. 2. UV intensity distribution. (a) Previous study [19]. (b) This study. The diameter of the image is $12 \mathrm{~mm}$.

A sapphire plate was used as a substrate instead of fused silica. The sapphire plate is almost transparent in the UV region. The refractive index of sapphire $(n=1.77)$ is larger than those of fused silica $(n=1.46)$ and UV curable resin $(n=1.48)$. Figure 3 shows a comparison of signals from the laser displacement sensor using fused silica and sapphire substrates.

As shown in Fig. 3(a), two peaks can be observed which originated from the reflection from the air/UV curable resin (1) and from the bottom of the substrate. For the sapphire substrate, the reflection from the substrate/UV-curable resin interface appeared as a $3^{\text {rd }}$ peak. The position of peaks (3) and (1) indicates the thickness of the sample. The thickness of the sample could be measured using the sapphire substrate. The film thickness was corrected using the refractive indexes of the UV curable resin and sapphire plate. 

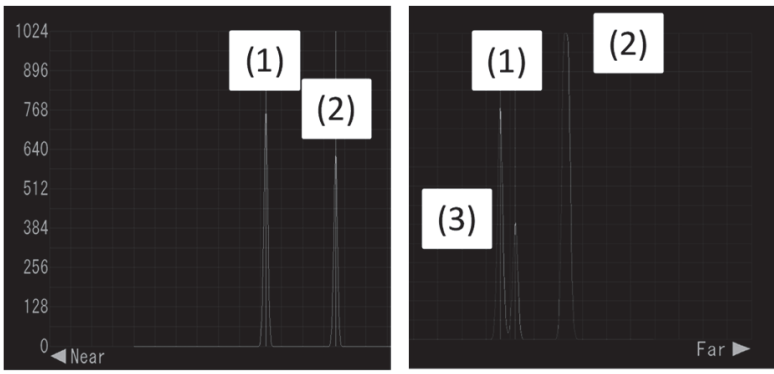

(a) Fused silica

(b) Sapphire

Fig. 3. Raw signals from the laser displacement sensor.

Two fused silica plates and a nitrogen-purging line were used to reduce the oxygen concentration. The nitrogen gas was supplied from a cylinder (purity $99.999 \%$ ) at a flow rate of $50 \mathrm{~mL} / \mathrm{min}$ for 20 min.

The measurement was conducted as follows: The sapphire plate $(\phi 24 \mathrm{~mm}, \mathrm{t} 0.3 \mathrm{~mm})$ was cleaned using Piranha solution. The UV curable resin was subsequently spun-cast on the sapphire plate. The rotation speed and casting time were adjusted to obtain a thickness of 50 to $130 \mu \mathrm{m}$. The sample was then placed on the measurement apparatus and allowed to settle for $20 \mathrm{~min}$. For measurements performed under the nitrogen atmosphere, the nitrogen was flushed after the sample was fixed and the two fused silica plates were fixed to the apparatus.

Measurement of the sample thickness was started prior to UV irradiation at $10 \mathrm{~Hz}$. After $10 \mathrm{~s}$, UV irradiation was performed for 3 s. During UV irradiation and post UV irradiation, measurement was continued. After $500 \mathrm{~s}$, the film thickness stabilized. Data points were obtained from 490 to $500 \mathrm{~s}$ and averaged to determine the final thickness. Similarly, data points from 0 to $10 \mathrm{~s}$ were averaged to calculate the initial thickness.

The initial and final thicknesses were measured as a function of UV intensity and atmosphere. The design and parameters of the experiment are listed in Table 1.

\section{Results and discussion}

Figure 4 shows the change of the film thickness during UV irradiation under nitrogen and air atmospheres. The UV intensity was $10 \mathrm{~mW} / \mathrm{cm}^{2}$ and irradiation time was $3 \mathrm{~s}$. The film thickness decreased upon UV irradiation. After the UV light was shut-off, the thickness decreased gradually until $500 \mathrm{~s}$. Radicals still exist in the air atmosphere, resulting in the continuation of polymerization and subsequent shrinkage.

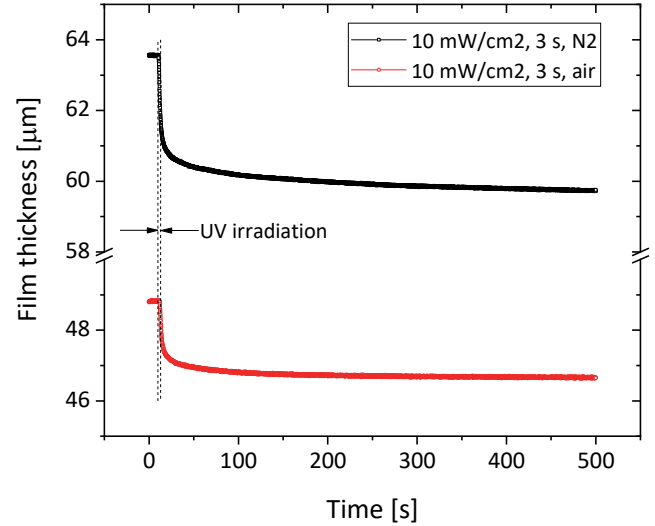

Fig. 4. Change in the film thickness during UV irradiation at an intensity of $10 \mathrm{~mW} / \mathrm{cm}^{2}$ for $3 \mathrm{~s}$.

The relationship between the initial and final thickness measured in nitrogen and air atmospheres are plotted in Figs. 5 and 6, respectively. As expected, the final thickness was smaller than the initial thickness. Interestingly, a linear relationship was observed where the final thickness was proportional to the initial thickness.

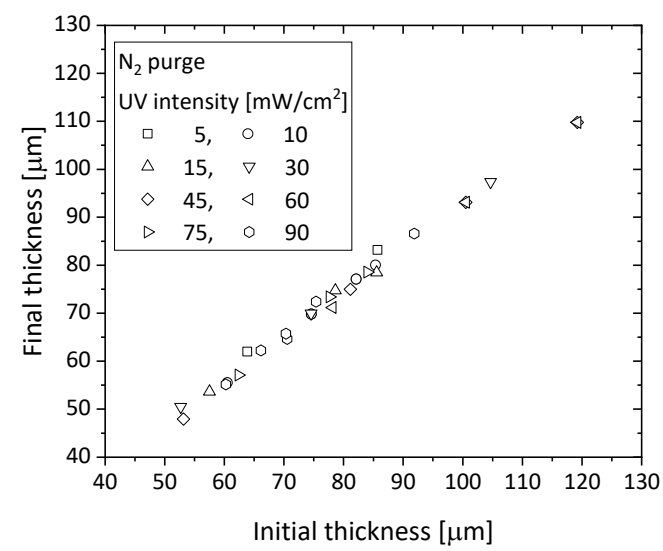

Fig. 5. Relationship between the initial and final thickness of the film UV-cured under a nitrogen atmosphere.

The slope and intercept of the linear relationship are listed in Table 1. The correlation coefficient, $r^{2}$, is greater than 0.99 , which indicates a good linear correlation was obtained between the final and initial thicknesses. The final thickness for an initial thickness of $80 \mu \mathrm{m}$, for instance, was calculated and the degree of shrinkage could also be obtained. This evaluation method for determining the degree of shrinkage is useful when the reproducibility of the initial thickness is relatively poor. 


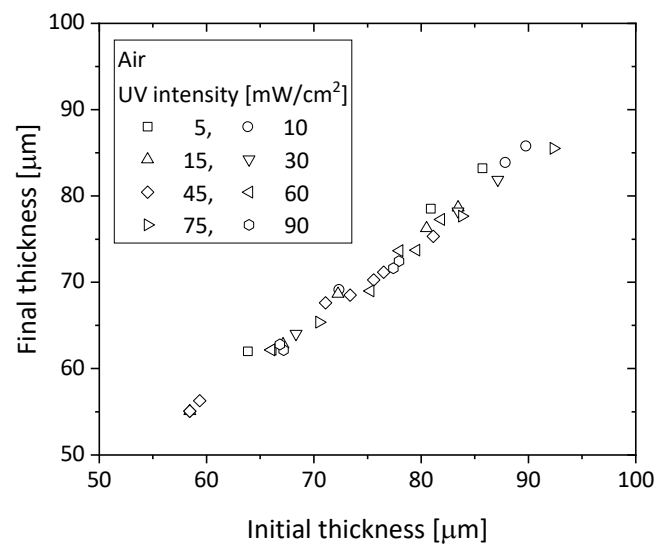

Fig. 6. Relationship between the initial and final thickness of the film UV-cured under an air atmosphere.

The effects of UV intensity and type of atmosphere on the degree of shrinkage are shown in Fig. 7.

The degree of shrinkage under a nitrogen atmosphere was determined to be larger than in air, as expected. The slope of the sample under nitrogen is constant from 10 to $100 \mathrm{~mW} / \mathrm{cm}^{2}$. The degree of shrinkage is $1.3 \%$ higher than in an air atmosphere. However, the slope of the sample in air exhibits a two-phase linear relationship, where the slope from 5 to $15 \mathrm{~mW} / \mathrm{cm}^{2}$ is larger than from 15 to 100 $\mathrm{mW} / \mathrm{cm}^{2}$. In a previous study, the same material and UV light source were used [23], and the thickness of the unreacted layer formed by oxygen inhibition was measured. The thickness of the unreacted layer decreased with increasing UV intensity. The slope of the unreacted layer as a function of UV intensity changed at $10 \mathrm{~mW} / \mathrm{cm}^{2}$. The unreacted layer thickness increased sharply with decreasing UV intensity. Although the UV intensity and the degree of shrinkage and unreacted layer were not in exact agreement, UV intensity below $10 \mathrm{~mW} / \mathrm{cm}^{2}$ formed a thick unreacted layer. As the unreacted layer does not shrink, the thickening of this layer decreased the degree of shrinkage.

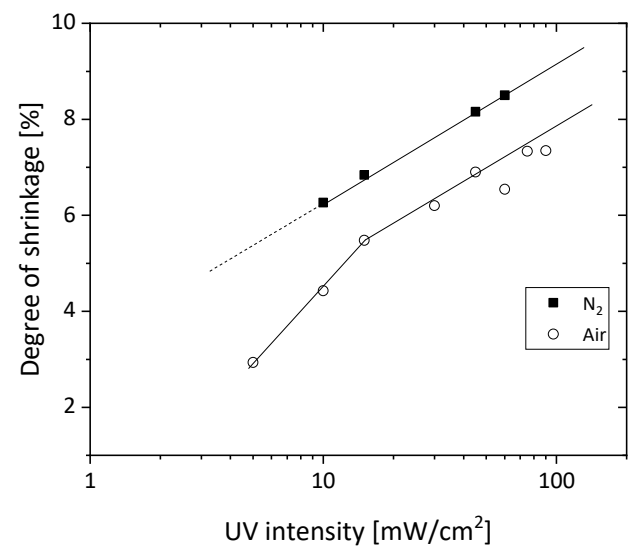

Fig. 7. Effect of the atmosphere on the degree of shrinkage of the UV-curable resin.

\section{Limitation}

It should be noted that this measurement technology has several limitations.

When the viscosity of the UV-curable resin is too low, the resin surface moves inhomogeneously during photopolymerization. In this apparatus, the UV light is applied at $\phi 12 \mathrm{~mm}$ of $\phi 24 \mathrm{~mm}$ of circular disk. When the UV light was applied to the center of the disk around $6 \mathrm{~mm}$, the area shrunk and the resin outside of the $\phi 12 \mathrm{~mm}$ flowed to the center. The film thickness decreased by shrinking and then increased from the flow of outside resin. This situation likely occurred in the low-viscosity UVcurable resin. To avoid the flow of resin, a metal

Table 1. Degree of shrinkage at different UV intensities and atmospheres.

\begin{tabular}{ccccccc}
\hline & $\begin{array}{c}\text { UV intensity } \\
{\left[\mathrm{mW} / \mathrm{cm}^{2}\right]}\end{array}$ & $\begin{array}{c}\text { Slope } \\
{[-]}\end{array}$ & $\begin{array}{c}\text { Intercept } \\
{[\mu \mathrm{m}]}\end{array}$ & $r^{2}$ & $\begin{array}{c}\text { Calculated final thickness* } \\
{[\mu \mathrm{m}]}\end{array}$ & $\begin{array}{c}\text { Degree of } \\
\text { shrinkage [\%] }\end{array}$ \\
\hline \multirow{4}{*}{$\mathrm{N}_{2}$} & 10 & 0.9525 & 0.2567 & 1.000 & 76.46 & 4.43 \\
& 15 & 0.9571 & -0.9515 & 0.999 & 75.62 & 5.48 \\
& 45 & 0.8875 & 3.4799 & 0.999 & 74.48 & 6.90 \\
& 60 & 0.9210 & 1.0843 & 0.991 & 74.76 & 6.54 \\
\hline \multirow{4}{*}{ Air } & 5 & 0.9694 & 0.1031 & 1.0000 & 77.66 & 2.93 \\
& 10 & 0.9905 & -4.2518 & 0.9998 & 74.99 & 6.27 \\
& 15 & 0.9120 & 1.5662 & 0.9949 & 74.53 & 6.21 \\
& 30 & 0.9025 & 2.8271 & 1.0000 & 75.03 & 8.16 \\
& 45 & 0.9382 & -1.5786 & 0.9998 & 73.47 & 8.50 \\
\hline
\end{tabular}

${ }^{*}$ Calculated final thickness was based on an initial thickness of $80 \mu \mathrm{m} .{ }^{* *}$ Degree of shrinkage $=1-($ Final thickness)/(Initial thickness). 
washer was placed on the center of sapphire plate, which effectively prevented the flow.

Another limitation is the calculation method of the degree of shrinkage. This study used the slope and intercept of the linear correlation of the final and initial thicknesses. When the degree of shrinkage was calculated, the initial thickness must set in a range where the final thickness could be calculated by interpolating the linear correlation. Extrapolation does not always give reasonable degrees of shrinkage.

\section{Conclusion}

The degree of shrinkage was determined by developing a novel laser displacement apparatus to monitor film thickness during UV curing. The apparatus consisted of an off-axis parabolic mirror for uniform UV intensity distribution, sapphire plate substrate for measuring exact film thickness, and nitrogen purge port with two fused silica windows for oxygen-free UV curing. The results indicated that the initial and final thicknesses exhibited a linear relationship. The effect of UV intensity on the degree of shrinkage was evaluated by calculating the final thickness for an initial thickness of $80 \mu \mathrm{m}$ using the slope and intercept of each linear relationship. Under an air atmosphere, the degree of shrinkage initially increased with the UV intensity steeply and subsequently increased gently. Under a nitrogen atmosphere, the degree of shrinkage increased monotonically.

Although the developed apparatus had some limitations, advances made here are crucial to developing multi-purpose thickness-measuring devices for determining the degree of shrinkage, conversion, and residual stress simultaneously. The comparison of the degree of shrinkage is important to predict shrinkage distributions in complex micromanufacturing processes.

\section{Acknowledgements}

This work was partly supported by the Die and Mould Technology Promotion Foundation (Japan) and the Grant-In-Aid of Japanese government for Challenging Exploratory Research (16K14469). We would like to thank Editage (www.editage.jp) for English language editing.

\section{References}

1. H. Lu, J. W. Stansbury, and C. N. Bowman, Den. Mater., 20 (2004) 979.
2. R. R. Braga, R. Y. Ballester, and J. L. Ferracane, Den. Mater, 21 (2005) 962.

3. S. Johnson, R. Burns, E. K. Kim, G. Schmid, M. Dickey, J. Meiring, S. Burns, N. Stacey, C. G. Willson, D. Convey, Y. Wei, P. Fejes, K. Gehoski, D. Mancini, K. Nordquist, W. J. Dauksher, and D. J. Resnick, J. Photopolym. Sci. Technol., 17 (2004) 417.

4. Y. Hirai, J. Photopolym. Sci. Technol., 23 (2010) 25.

5. M. Shibata, A. Horiba, Y. Nagaoka, H. Kawata, M. Yasuda, and Y. Hirai, J. Vac. Sci. Technol. B Nanotechnol. Microelectron., 28 (2010) C6M108.

6. R. Suzuki, N. Sakai, A. Sekiguchi, Y. Matsumoto, R. Tanaka, and Y. Hirai, J. Photopolym. Sci. Technol., 23 (2010) 51.

7. A. Amirsadeghi, J. J. Lee, and S. Park, Langmuir, 28 (2012) 11546.

8. M. Dehurtevent, L. Robberecht, J. C. Hornez, A. Thuault, E. Deveaux, and P. Béhin, Den. Mater., 33 (2017) 477.

9. G. Bugeda, M. Cervera, G. Lombera, and E. Oñate, Rapid Prototyp. J., 1 (1995) 13.

10. W. L. Wang, C. M. Cheah, J. Y. H. Fuh, and L. Lu, Mater. Des., 17 (1996) 205.

11. Y. M. Huang, Int. J. Adv. Manuf. Technol., 21 (2003) 586.

12. K. Taki, Y. Watanabe, T. Tanabe, H. Ito, and M. Ohshima, Chem. Eng. Sci., 158 (2017) 569.

13. C. N. Bowman and N. A. Peppas, Macromolecules, 24 (1991) 1914.

14. K. S. Anseth, C. N. Bowman, and N. A. Peppas, J. Polym. Sci. A-Polym. Chem., 32 (1994) 139.

15. K. S. Anseth, L. M. Kline, T. A. Walker, K. J. Anderson, and C. N. Bowman, Macromolecules, 28 (1995) 2491.

16. D. C. Watts, A. S. Marouf, and A. M. Al-Hindi, Den. Mater., 19 (2003) 1.

17. H. Lu, J. W. Stansbury, S. H. Dickens, F. C. Eichmiller, and C. N. Bowman, J. Biomed. Mater. Res. Part B, 71B (2004) 206.

18. W. K. Neo and M. B. Chan-Park, Macromol. Rapid Commun., 26 (2005) 1008.

19. Y. Jian, Y. He, T. Jiang, C. Li, W. Yang, and J. Nie, J. Polym. Sci. B: Polym. Phys., 50 (2012) 923.

20. C. Schmidt and T. Scherzer, J. Polym. Sci. B: Polym. Phys., 53 (2015) 729.

21. G. F. Arenas, M. N. Guzmán, C. Vallo, R. Duchowicz, and M. Trivi, Polym. Test., 50 (2016) 262.

22. S. C. Ligon, B. Husár, H. Wutzel, R. Holman, and R. Liska, Chem. Rev., 114 (2013) 557.

23. K. Taki and T. Nakamura, J. Cosmet. Dermatolog. Sci. Appl., 1 (2011) 111. 